\title{
Video-based assistance system for training in minimally invasive surgery
}

\author{
JUAN A. SÁNCHEZ-MARGALLO ${ }^{1}$, FRANCISCO M. SÁNCHEZ-MARGALLO ${ }^{1}$, \\ JOSÉ B. PAGADOR ${ }^{1}$, ENRIQUE J. GÓMEZ ${ }^{2}$, PATRICIA SÁNCHEZ-GONZÁLEZ ${ }^{2}$, \\ JESÚS USÓN ${ }^{1}$, JOSÉ MORENO ${ }^{3}$
}

${ }^{1}$ Minimally Invasive Strgery Centre fesús Usón, Cáceres, Spain, ${ }^{2}$ Biomedical Engineering and Telemedicine Centre, Polytechnic Universiry of Madrid, Madrid, Spain, and ${ }^{3}$ Laboratory of Robotics and Arificial Vision, University of Extremadura, Cáceres, Spain

\begin{abstract}
In this paper, the development of an assisting system for laparoscopic surgical training is presented. With this system, we expect to facilitate the training process at the first stages of training in laparoscopic surgery and to contribute to an objective evaluation of surgical skills. To achieve this, we propose the insertion of multimedia contents and outlines of work adapted to the level of experience of rrainees and the detecrion of the movements of the laparoscopic instrument into the monitored image. A module to rrack the instrument is implemented focusing on the tip of the laparoscopic tool. This rracking method does not need the presence of artificial marks or special colours to disringuish the instruments. Similarly, the system has another method based on visual tracking to localize support multimedia content in a stable position of the field of vision. Therefore, this position of the support content is adapted to the movements of the camera or the working area. Experimental results are presented to show the feasibility of the proposed system for assisting in laparoscopic surgical training.
\end{abstract}

Key words: Laparoscopy, laparoscopic instrument tracking, training, visual tracking, video-based system

\section{Introduction}

The introduction of laparoscopic surgery constitutes one of the most important advances in the field of surgery in the last twency years, and it scill has a great impact on surgical practice. This surgical procedure has modified many well-established surgical concepts, such as the reduccion of surgical stress $(1,2)$, less parietal problems (3), reduction of post-operative pain (4), better aesthetic results (5), and faster reincorporation into the working life $(6-8)$, and as consequence the sanitary $\cos t s$ are reduced. These benefits have led to many surgeons being trained in this surgical procedure (9).

In order to carry out an adequate performance of laparoscopic surgery it is important to progress from the basic principles to advanced skills through a steep learning curve (10). However, future advances should improve these practical aspects of training in laparoscopy (11).

In laparoscopic surgery the video images acquired from the laparoscopic camera are displayed in real time on a monitor to provide visual feedback to the surgeon (12), thus minimally invasive procedures guided by imaging involve a new paradigm in surgical procedures and surgical training.

Regarding the training process in laparoscopic surgery, especially during the early stages, teachers have some difficulties such as paying attention to all trajnees simultaneously, repeating the same contents of explanation and making a subjective assessment. We hypothesize that this raining process in laparoscopic surgery could be improved by providing videobased assistance during the performance of surgical tasks and a real-cime tracking method applied to laparoscopic instruments. Therefore, this racking

Correspondence: J. A. Sánchez Margallo, Minimally Invasive Surgery Centre Jestís Usót, Ctra. 10071 Cáceres, Spain. Fax: +34927181033.

E-mail: jasanchez@cmijesususon.com 
method allows us to carry out an objecrive assessment of surgical skills.

To make the training in laparoscopic surgery during the early stages easier, there are currently several hytrid simulation systems. Most of them are focused on the objective assessment of surgical skills (12-15). Others focus on providing an image more similar to a real surgical scenario. Yet others include the insertion of virtual contents into the training process (16).

Regarding the tracking of the laparoscopic instruments, several approaches have been proposed to deal with this problem. We think that addressing the problem by using image processing techniques to track the inscrument is an interescing alternative to others based on the use of sensors located on the instrument (optical, electromagnetic or mechanical) which can be very uncomfortable and unmanageable for surgeons. In the scientific literature, some of these approaches analyze the colour of the instrument as Wang et al. (17), who carry out a statiscical analysis to discinguish between pixels belonging to a laparoscopic inscrument or environment of the abdominal cavity. Other authors (18), however, perform statiscical analysis using Bayes theorem. On the orher hand, Doignon et al. (19) use an image segmentation to find the instrument using the hue and saturation of the image with a HSI (Hue, Saturation, Intensicy) colour model and region growing techniques.

In works that use artificial marks on the laparoscopic instruments, these marks are often located next to the rip and then are identified by image segmentacion. The use of an unusual colour in surgical images, as arcificial mark, is performed in orher cases $(20,21)$. Zhang and Payandeh (22) use a pattern formed by three black lines with the same width and space. Tonet et al. (23) propose a heuristic approach to estimate the depth of the instrument by means of the knowledge of the instrument width and the orientation of its edges. Others propose the use of a LED placed in the tip of the instrument that projects laser doss onto the surface of organs to allow them to localize the instrument with respect to the scene (24).

Another possibilicy is not using artificial marks or analyzing the colour of the instrument. For instance, Climent and Marés (25) and Voros et al. (26) employ the Hough transform to find the straight lines belonging to the edges of the instrument. Besides using the Hough transform, Voros et al. (26) also use the Otsu thresholding and the position of the insertion point of the instrument to restrict the search range of the tool. On the other hand, other authors (27) propose a geomerrical opcical model to determine the rip position and the orientation of a laparoscopic instrument with respect to the camera coordinates.
In this paper, a new way to improve the laparoscopic training process for new trainees is presented. This system supports the surgical rraining with videobased contents (image and video) and carries out an automatic vision-based tracking of the movement performed by the laparoscopic instruments. This tracking method could be useful, in future works, to analyze the movements performed by trainees and make an objecrive assessment of surgical skills during the surgical training process.

\section{Material and methods}

To standardize the performance within a controllable and reproducible scenario, we use the physical laparoscopic simulator SIMULAP-IC05 ${ }^{\text {(2) }}$ (CCMIJU, Cáceres, Spain) $(9,28,29)$, mainly dedicated to the learning of laparoscopic maneuvers which involve some technical difficulcy. This training device simulates the abdominal cavicy and presents a transparent cover made of plastic, which allows the insercion of the trocars for the handling of the laparoscopic instruments. The surgeon follows his maneuvers performed through the displayed images on the screens.

For the implementation of the system, we use the $\mathrm{C}++$ programming language and the OpenCV 1.0.1 graphic library (30), all under a Linux-based operating system.

The video-based system is implemented on a computer with an Intel ${ }^{\text {Q }}$ Core $^{\mathrm{TM}} 2$ processor to $2.4 \mathrm{GHz}$ and 3GB RAM. The system has a standard video capture card, a NVidia GeForce GTX $260896 \mathrm{MB}$ DDR3 graphic card and a TFT monitor which has 17 inches, $1024 \times 768$ resolution, $500 \mathrm{~cd} / \mathrm{m}^{2}$ brightness, $400: 1$ contrast, $0.297 \mathrm{~mm}$ of pixel size and $16 \mathrm{~ms}$ response time.

The image capture from the camera of the physical simulator is taken by a RCA video connector connected to the video capture card of the computer.

The hardware components of the system are installed in a tower (Figure 1), which integrates the physical simulator, the corresponding camera, a screen to display the informacion and a computer which captures the video image and runs the processes of the assisting system.

\section{Visual tracking for the viewing support content}

First a method which performs a real-cime visual tracking of one point or area of the image from the laparoscopic camera is developed. This visual tracking method facilitates the superposition on this point of support visual content: A static image (i.e., an outline of the tasks), or an embedded video object. 


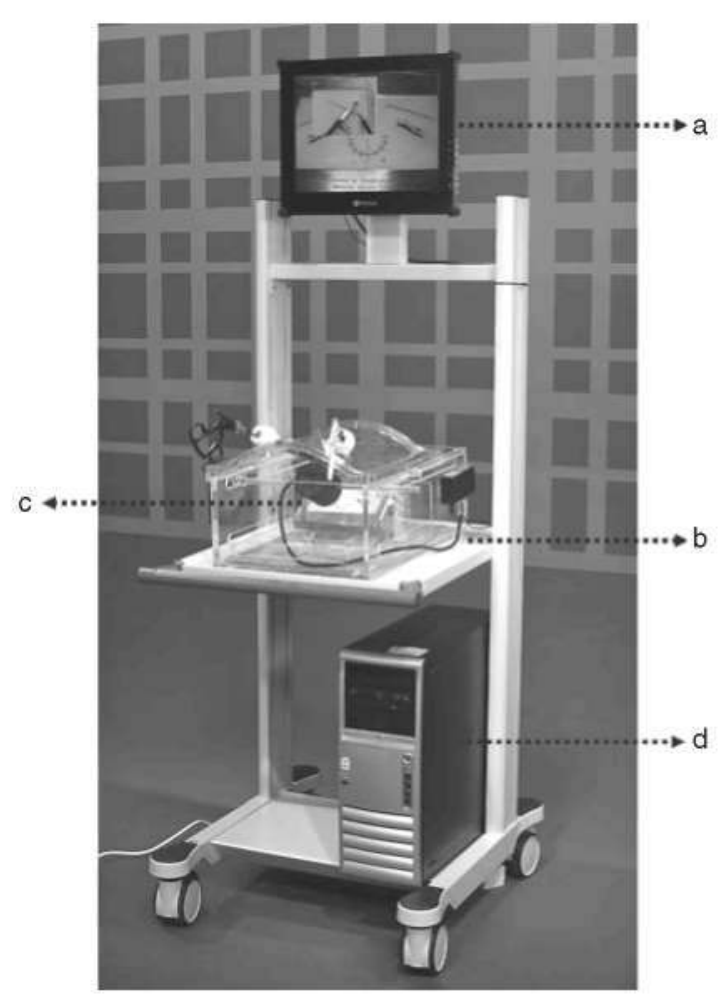

Figure 1. The complete system consists of a monitor (a), the physical laparoscopic simulator (b), a camera (c), and a computer (d).

This visual tracking method performs an analysis of the optical flow via the Lucas-Kanade algorithm with Gaussian Pyramids (31,32). This algorithm accomplishes the extraction of the $x$ and $y$ components of the velocity vector, in a local neighborhood around the analyzed point. Therefore, an estimation of the optical flow in blocks of pixels is established, and thus the movement vector for this block is considered.

The pyramidal Gaussian representation of an image consists of creating a series of images, so that each image is the Gaussian distribution of its previous image. Thus, the representation of the image is divided into different hierarchical levels. The first level is the original image, the second one is the image after applying it to a Gaussian filter, and so on.

The aim of this visual tracking method is to determine the position of a certain point of an image in the next frame of the video sequence. The method keeps the superimposed visual content in a stable position of the working area.

A pyramidal representation ( $n$ levels) is obtained from two consecutive images of the sequence; being $n$ the level with more Gaussian smoothing, and 0 the initial level (original image). In our particular case, we use five levels for the pyramidal representation of the Gaussian image.
For each level, starting from the $n^{\text {th }}$ level backwards, using the two consecutive images of the sequence, the velocity vector of the selected point is calculated by the Lucas-Kanade optical flow method. For one point of the $i^{\text {th }}$ level $(1<i<n)$ corresponds to a block of points of level $i-1$ of the pyramidal representation. Therefore, if we have the velocity vector of the $i^{\text {th }}$ level point (calculated in the previous step), we will obtain the velocity vector of a block of points in the level $i-1$. When we reach the last level, we have the motion estimation of the marked point in the original image (level 0 ).

Once we track the marked point in the current frame, the next step is to establish the region of interest (ROI) superimposed on this point, so the support visual content is inserted within this ROI. We define the rectangular structure of the ROI with the same width and height as the visual content and with the same coordinates of its centre as the tracked point in the image. Now, we can insert a static image, outlines of the laparoscopic task or a video tutorial into this region.

This visual tracking method updates the position of the visual content each time there are changes in the sequence of video which affect the position of the tracked point. Therefore, the visual content is always located in a stable position of the working area in the field of vision.

For assessment, we take three 25 -second video sequences, each one of them with a marked point to which is applied the tracking algorithm. For each video sequence, we carry out different movements of the camera to analyze the behavior of this method: Horizontal, vertical and diagonal movements.

For each 25-second video sequence, we take one frame per second ( 25 frames in total) and an expert evaluates manually the location of the marked point within the limits in a neighborhood of 24 pixels, which is considered unnoticeable for the user. This threshold was empirically determined, so that 24 pixels of neighborhood were ideal for the given images sequences.

\section{Laparoscopic instrument tracking method}

An automatic classifier system is developed, previously trained, to be able to distinguish among images where our object of interest appears and images where it does not, and its position in the image.

The classifier is developed in four phases: Image acquisition, creation of samples, training, and testing.

The first step is to take the images to be used to train the classifier. These images are of two kinds: Negative and positive (Figure 2). The negative images 

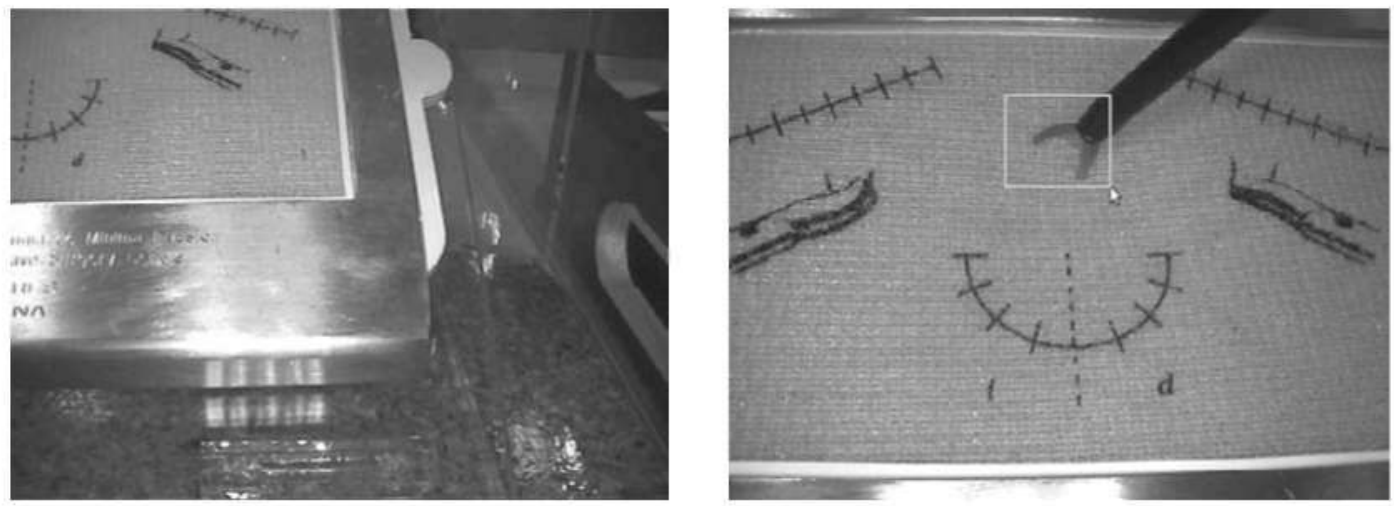

Figure 2. Negative image (left). Positive image (right), selection of the coordinates and dimensions of the object of interest (tip of the laparoscopic scissors).

are taken in an arbitrary way, and they do not contain the object to be identified. The positive images contain the object to be identified, and it is necessary to indicate the coordinates and dimensions of this object. In the positive images, the object must appear in a clear way, in different angles, in different backgrounds, and with different illumination settings. The positive and negative images are exclusively acquired inside of the physical simulator. To facilitate the task of selecting the coordinates and the dimensions of the objects, we develop a marking application tool which allows users to use the mouse for this purpose.

To proceed to the creation of samples, the vector which contains the sample images (standardized, grayscale and proper-sized) is created.

The following stage is the training of the classifier. We use the OpenCV graphic library to carry out a training based on AdaBoost algorithm (33). AdaBoost is a learning algorithm focused on two important aspects: Selection of a set of features which represent the object of interest, and training the classifier with a lineal combination of the best features.

When the training stage is over, we have a cascade classifier (34); this classifier points out if there are any tips of laparoscopic instruments in the input image. In case the algorithm gives a positive answer, we will know in real time the position of the tip of the laparoscopic tool in the image.

Regarding assessment, the tests are tried on three 25 -second video sequences. Conditions of these sequences vary in terms of illumination, speed of movement and position of the instrument in the field of vision.

For each sequence, we take one frame per second (25 frames in total) and an expert manually assesses the result of the automatic method for detecting the tip of the analyzed laparoscopic instrument, using laparoscopic scissors.

\section{Results}

Measuring of the effectiveness of the methods allows us to determine the validity of the system for assistance during the training of basic laparoscopic tasks.

The results of the reliability of the visual tracking method (Figures 3 and 4) are presented in three different graphs with regard to horizontal (Figure 5), vertical (Figure 6) and diagonal (Figure 7) movements of the camera.
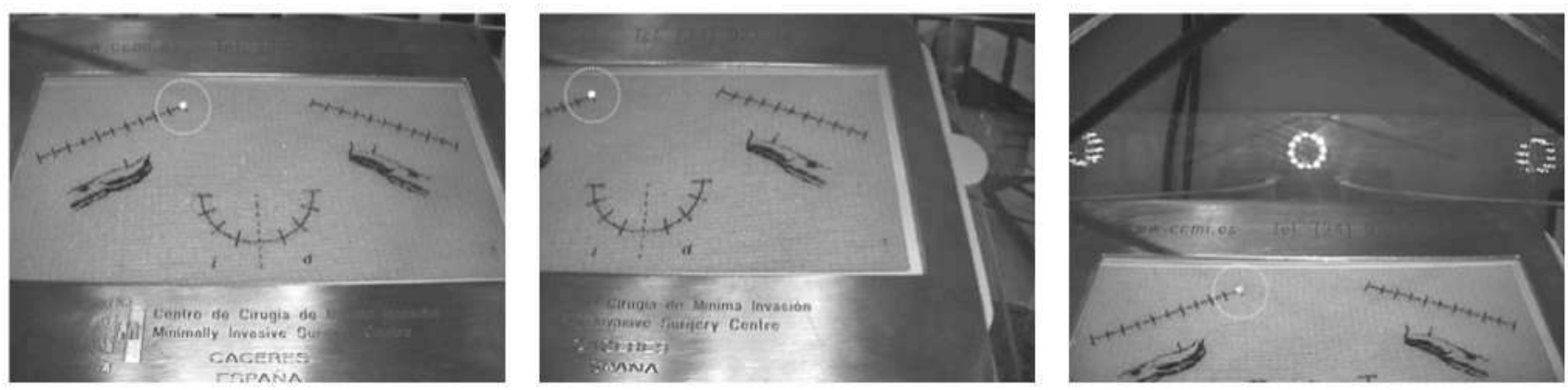

Figure 3. The visual tracking method in a sequence of images. The method is applied to a point previously selected by the user (in white and highlighted with a dashed circle) of the working area (latex sheet) and with different movements of the camera. 

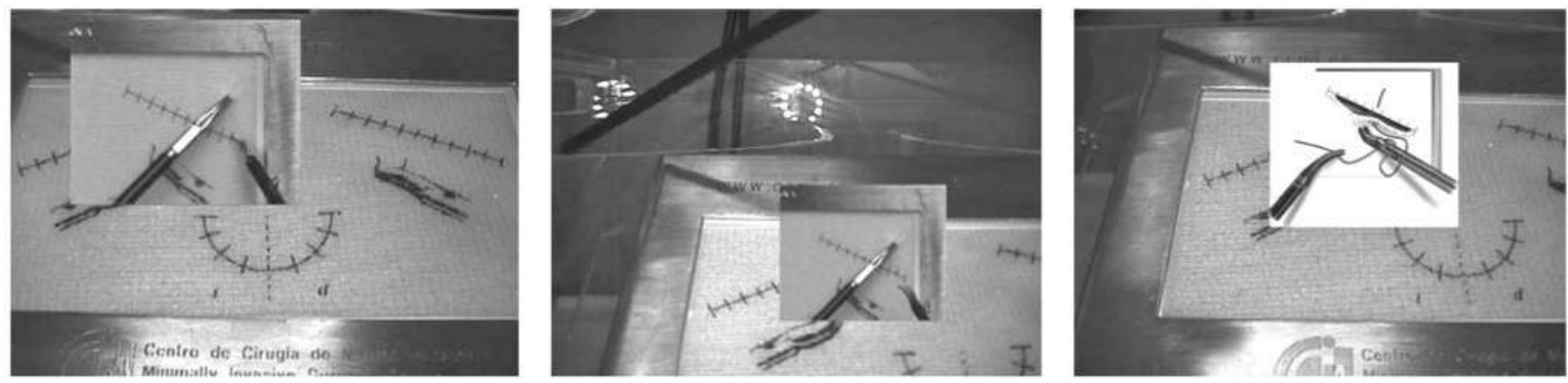

Figure 4. Three sequences of laparoscopic tasks which include several support visual contents, such as a video sequence (left and center images) and an outline of the task (right image). The support content remains in a stable position with respect to the movements of the camera and working area.

The result will be considered wrong if the marked point is located outside a neighbourhood of 24 pixels from its real position. Therefore, the results show that the horizontal movements of the camera (Figure 5) provide better results ( $84 \%$ success) for the visual tracking method than vertical (Figure 6) (72\% success) and diagonal movements (Figure 7) (64\% success). We consider that results with success rates near to $100 \%$ provide greater robustness to the system under different working conditions. Taking into account the results obtained, they show that diagonal movements of the camera make it difficult to predict the correct position of the marked point by this visual tracking method. The percentage of success is calculated by dividing by the number of success cases and the number of analyzed frames.

The results of the tracking method of the tip of the laparoscopic scissors (Figure 8) are summarized in

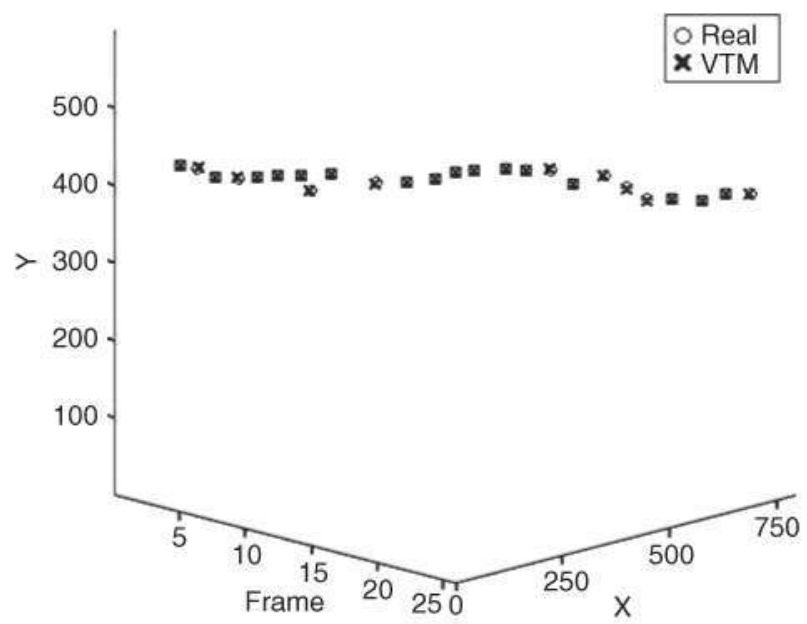

Figure 5. Recorded position ( $\mathrm{x}, \mathrm{y})$ of the marked point along 25 frames of a video sequence (frame) in which a horizontal movement with the camera is performed. The graph shows the position obtained by the visual tracking method (VTM) and the real position which should be obtained (real). the Table I. Three sequences of images with different working conditions are analyzed: For Sequence 1, the speed of movements is increased and the usual positions of the scissors in the working area are avoided. For Sequence 2, a usual laparoscopic suturing task is carried out under usual working conditions (illumination, speed of movements and position of the scissors). Finally, for Sequence 3, the usual light conditions in the laparoscopic simulator are decreased.

As observed, in Sequence 3, the lack of illumination can worsen the process of recognition of the object. On the other hand, for Sequence 1, the movements of the scissors are faster and are mainly located in areas of the field of vision with texture and colour similar to the tip of the laparoscopic instrument, such as the metallic edge of the working sheets holder. Consequently, both circumstances complicate the identification of the tip of the scissors.

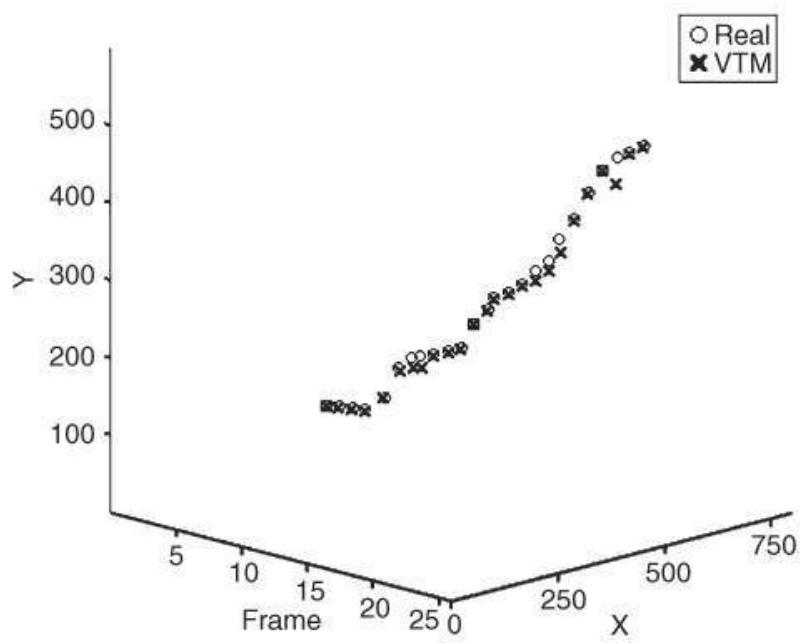

Figure 6. Recorded position ( $\mathrm{x}, \mathrm{y})$ of the marked point along 25 frames of a video sequence (frame) in which a vertical movement with the camera is performed. The graph shows the position obtained by the visual tracking method (VTM) and the real position which should be obtained (real). 


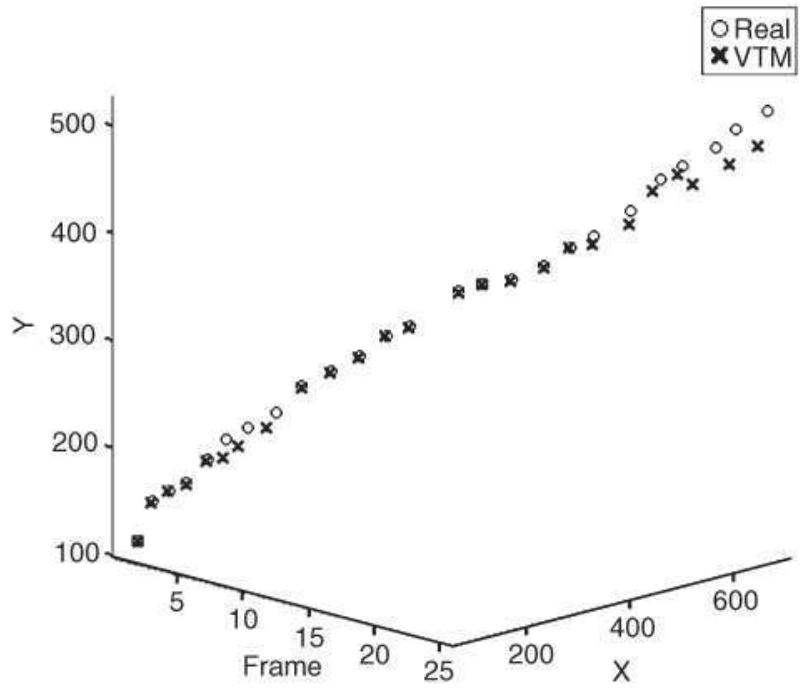

Figure 7. Recorded position ( $\mathrm{x}, \mathrm{y}$ ) of the marked point along 25 frames of a video sequence (frame) in which a diagonal movement with the camera is performed. The graph shows the position obtained by the visual tracking method (VTM) and the real position which should be obtained (real).

\section{Discussion}

It has been widely reported that laparoscopic surgery offers a lot of benefits for the patient in comparison to open surgery, and these benefits have led to the need that many surgeons are trained in this surgical procedure. However, future advances should improve some practical aspects of training in laparoscopy.

Due to the surgeon's need for visual feedback to perform the surgical training tasks, we hypothesize that this training process could be improved by providing video-based assistance using support visual contents (video tutorial or outline of the task) during surgical tasks and adapted ones to the level of experience of trainees. Similarly, analyses of path and movements travelled by laparoscopic instruments and their speed are important sources to carry out an objective evaluation of surgical skills (35).

This paper presents the first steps in developing a new hybrid simulation system. This system has the advantages of the physical laparoscopic simulator SIMULAP-IC0 ${ }^{(1)}$ (CCMIJU, Cáceres, Spain) and combines the performance of laparoscopic tasks with the use of computer vision techniques.

Preliminary results show satisfactory percentages of success $(84 \%, 72 \%$ and $64 \%)$ (Figure 5,6 and 7 ) with regard to the assessment of the visual tracking method and prove the effectiveness of the system for localization of support video-based content. Likewise, the favorable results (success rates of $72 \%, 88 \%$ and $76 \%$ ) (Table I) with respect to the detection of the tip of the laparoscopic scissors show that the system is appropriate for the analysis of the movement of this instrument and for later use with another type of laparoscopic instrument.
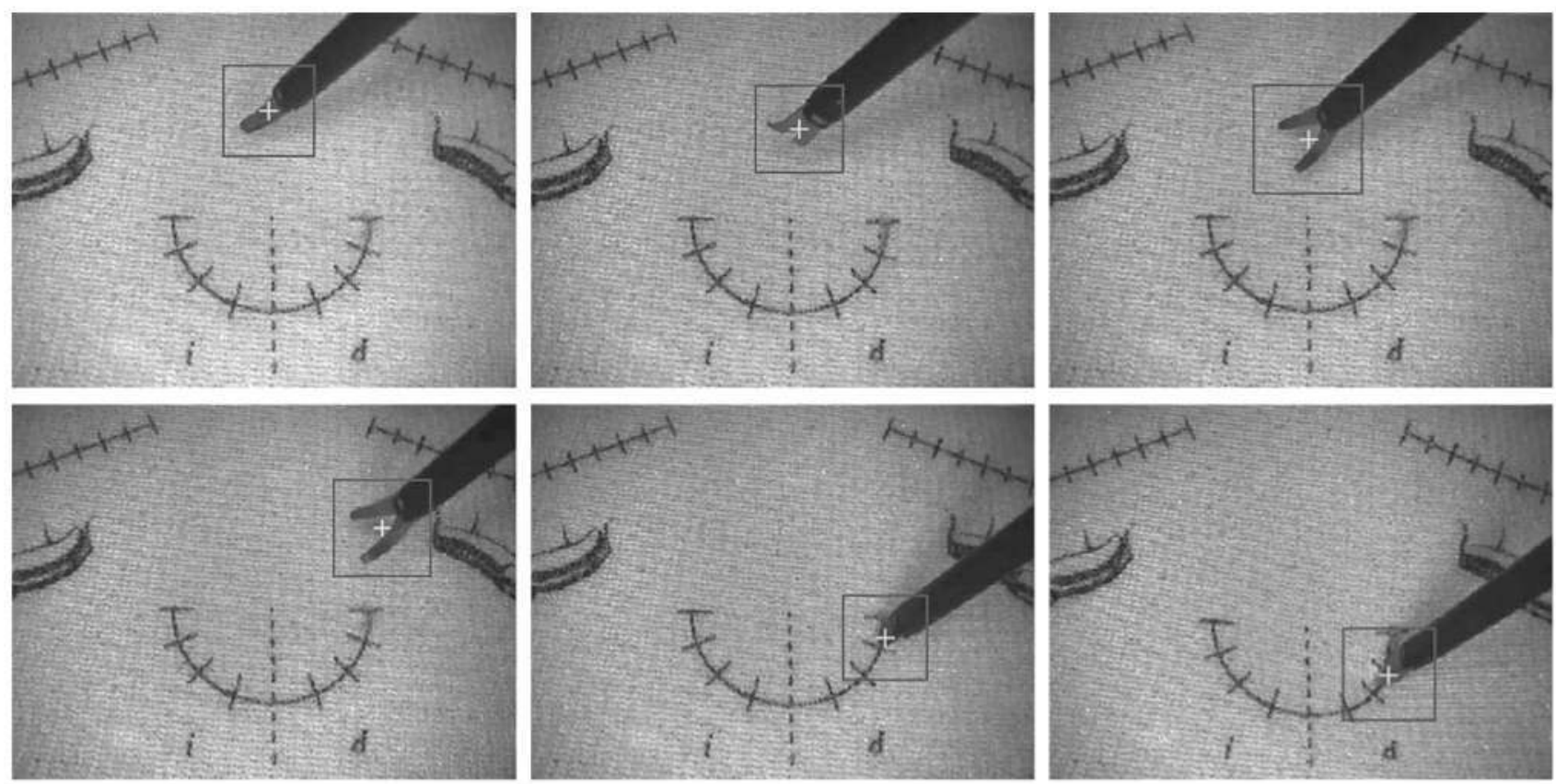

Figure 8. Example of execution of the automatic tracking method of the tip of the laparoscopic scissors. The sequence of six images shows the bounding boxes of the tracking method of the tip of the laparoscopic instrument. This sequence of images corresponds to a task of cutting a sheet of latex with laparoscopic scissors. 
Table I. Resuls of the tracking of the laparoscopic scissors tip in three sequences of images.

\begin{tabular}{lccc}
\hline & Sequence 1 & Sequence 2 & Sequence 3 \\
\hline Images (frames) & 25 & 25 & 25 \\
No. Successes & 18 & 22 & 19 \\
No. Mistakes & 7 & 3 & 6 \\
$\%$ Successes & $72 \%$ & $88 \%$ & $76 \%$ \\
\hline
\end{tabular}

Nowadays, there are several hybrid systems for training in laparoscopic surgery. One of these systems is the laparoscopic simulator CELTS ${ }^{(9)}$ (Center for Integration of Medicine and Innovative Technology CIMIT, Cambridge, MA, USA) (12), which incorporates two haptics mechanisms to analyze the movements of each of the laparoscopic instruments used. Other types of hybrid simulators are the

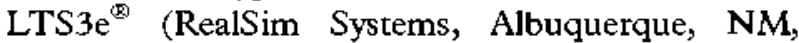
USA) (13) and SurgicalSim-LTS ${ }^{\infty}$ (METI, Sarasota, FL, USA) (14), which implement a series of tests using a set of sensors for measuring the efficacy during the performance of laparoscopic craining tasks. The laparoscopic simulator with SimuVisio ${ }^{\text {(S) }}$ (SimuLab Corporation, Seattle, WA, United States) only focuses on improving the quality of the displayed image to convert it into a more real one. On the other hand, an ultrasound system for locating laparoscopic instruments is used by Zebris ${ }^{(\mathbb{1}}$ (Zebris Medical GmbH, Isny, Germany) (15). Finally, somewhat more advanced is the laparoscopic simulator ProMIS $^{\oplus}$ (Haprica Inc., Boston, MA, USA) (16), which makes use of augmented reality techniques and performs the insertion of virtual contents together wich real objects.

Besides sharing some of the features of the mentioned hybrid systems, the proposed system offers the possibility of insercing support visual contents adapted to the level of experience of the trainees and allows them to determine the localization of these contents on the field of vision in a stable way. As a result, by employing visual tracking techniques for points or areas of the image, the contents are visualized and localized wirhin these areas, and suffer no displacement due to possible movements of the camera or objects of the working area. In this way, possible interruptions in the field of vision of the working area are avoided.

The proposed system uses a cascade classifier (34), previously trained, to automatically identify the position of the tip of the laparoscopic instrument in a standardized surgical environment with different setcings.

Regarding the results of the behavior analysis of the visual tracking mechod, it is possible that the increase in the number of mistakes is mainly due to the kind of movements developed by the camera. Diagonal movements of the camera are more complex than horizontal and vertical movements, and make the calculation of the motion estimation of the marked point in the image difficult.

As regards the results of the laparoscopic instrument racking method, rapid movements of laparoscopic scissors, lack of illuminacion, and the locacion of the instrument in areas with background texture and color similar to its ip increase the number of mistakes during the tracking process.

One way to decrease the rate of mistakes is to include a larger set of training cases for the classifier, increasing the number of cases with lack of illumination and situations where the tip of the laparoscopic instrument is localized in areas which present difficulties for its identification.

The method used for tracking the laparoscopic scissors has permitted the system to work without colour restriccions or special marks on the instrument, in contrast to many reports in the scientific literature. The system is not affected by the specular reflections as ocher approaches implement some mechods to solve this problem $(17,22,26)$.

Total occlusion of the tip of the laparoscopic instrument complicates the detection process of this imageguided system. Nevertheless, some studies partially solve this problem by estimating the instrument position in successive frames.

This system allows real-time execurion to assist training in laparoscopic surgery. Consequently, the system may be used togecher wich real-cime objecrive mechods to assess a surgeon's skills, wich automaric positioning methods of the laparoscopic camera or with augmented reality tools. Other studies leave open the possibility of the reduction of the speed of calculation of their methods, in order to achieve real-time execution $(26,27)$.

Like other studies, the tracking mechod of this system uses the $x$ and $y$ values to describe the cip of the laparoscopic inscrument, therefore the next step of this work is to calculate the depch of the rip of the instrument. We consider that depth value is important to complete the required information to carry out a proper objective assessment. To obtain this depth value, we propose the use of stereoscopic techniques (36) or geometrical optical models. In the same way, the widening of possible laparoscopic instruments that can be detected by the system is something to have in mind for future works.

A system to assist rraining in laparoscopic surgery was presented. This system performs assistance during the rraining process with the support of multimedia content adapted to the laparoscopic procedure 
and the level of experience of the trainees. This support content is located in a stable position of the field of vision. For extraccing the movement of the laparoscopic instrument, a robust visualbased tracking method of the instrument was implemented and presented. This method does not need the presence of artificial marks, or special colours to distinguish the tip of the instrument used. Similarly, this rracking mechod could be useful, in future works, to analyze the movernents performed by trainees and to make an objeccive assessment of surgical skills during the surgical training process.

\section{Acknowledgements}

This study was supported in part by grant PRI07B132 from Plan Regional de Investigación, Consejería de Economía, Comercio e Innovación, Junta de Extremadura and the European Social Fund. The auchors are especially grateful to Ms Elena Crespo for her technical assistance.

Declaration of interest: The auchors report no conflicts of interest. The authors alone are responsible for the content and writing of the paper.

\section{References}

1. Schiecroma $M$, Carlei $F$, Cappelli $S$, Pescosolido A, Lygidakis NJ, Anicucci G. Effects of cholecystectomy (laparoscopic versus open) on PMN-elastase. Hepatogastroenterology 2007;54:342-5.

2. Buunen $M$, Gholghesaei $M$, Veldkamp $R$, Meijer $D W$, Bonjer HJ, Bouvy ND. Suress response to laparoscopic surgery - A review. Surg Endosc. 2004;18:1022-8.

3. Beldi $G$, Ipakcchi $R$, Wagner $M$, Gloor $B$, Candinas D. Laparoscopic ventral hernia repair is safe and cost effecuive. Surg Endosc. 2006;20:92-5.

4. Cordera F, Long KH, Nagorney DM, McMurtry EK, Schleck C, Ilscrup D, et al. Open versus laparoscopic splenectomy for idiopathic thrombocytopenic purpura: Clinical and economic analysis. Surgery 2003;134:45-52.

5. Seizz G, Seizz EM, Kasparek MS, Konigsrainer A, Kreis ME. Long-term quality-of-life after open and laparoscopic sigmoid colectomy. Surg Endosc. 2008;18:162-7.

6. Delaney CP, Chang E, Senagore AJ, Broder M. Clinical outcomes and resource utilization associated with laparoscopic and open colectomy using a large nacional database. Ann Surg. 2008;247:819-24.

7. Roumm AR, Pizzi L, Goldfarb NI, Cohn H. Minimally invasive: minimally reimbursed? An examination of six laparoscopic surgical procedures. Surg Innov, 2005;12:261-87.

8. Nguyen $N T$, Zainabadi $K$, Mavandadi $S$, Paya $M$, Stevens $\mathrm{CM}$, Root $\mathrm{J}$, et al. Trends in utilization and outcomes of laparoscopic versus open appendeccomy. An J Surg. 2004; 188:813-18.

9. Usón J, Sánchez FM, Pascual S, Climent S. Formación en Cirugía Laparoscópica Paso a Paso. 3rd ed. Minimally Invasive Surgery Centre Jesús Usón, editor. Cáceres, Spain; 2007.
10. Sánchez-Margallo FM, Asencio JM, Tejonero MC, Sánchez MA, Pérez FJ, Usón J, et al. Training design and improvement of technical skills in the transvaginal cholecysrectomy (NOTES). Cir Esp. 2009;85:307-13.

11. Van Velthoven RF, Piechaud PT. Training centers: an essential step to developing skills in urolaparoscopy. Curr Urol Rep. 2009;10:93-6.

12. Stylopoulos $\mathrm{N}$, Corin $\mathrm{S}$, Dawson $\mathrm{S}$, Ottensmeyer $\mathrm{M}$, Neumann P, Bardsley $R$, et al. CELTS: a clinically-based Computer Enhanced Laparoscopic Training System. Stud Health Technol Inform. 2003;94:336-42.

13. Soyinka A, Schollmeyer $T$, Meinhold-Heerlein I, Gopalghare D, Hasson H, Mettler L. Enhancing laparoscopic performance with the LTS3e: a computerized hybrid physical reality simulator. Fertil Steril. 2008;90:1988-94.

14. Marhis $\mathrm{KL}$, Wiegmann DA. Construct validacion of a laparoscopic surgical simulator. Simul Healthc. 2007;2:178-82.

15. Sokollik C, Gross J, Buess G. New model for skills assessment and training progress in minimally invasive surgery. Surg Endosc. 2004;18:495-500.

16. Van Sickle $K R$, McClusky DA, Gallagher AG, Smith CD. Construct validation of the ProMIS simulator using a novel laparoscopic sucuring task. Surg Endosc. 2007;19:1227-31.

17. Wang YF, Uecker DR, Wang YL. A new framework for vision-enabled and robotically assisted minimally invasive surgery. Comput Med Imaging Graph. 1998;22:429-37.

18. McKenna $S$, Charif $H$, Frank $T$, editors. Towards Video Understanding of Laparoscopic Surgery: Inscrument Tracking. Proceedings of Image and Vision Computing; 2005; New Zealand.

19. Doignon $C$, Graebling P, Mathelin M. Real-time segmentarion of surgical instrumens inside the abdominal cavicy using a joint hue saturation color feature. Real-Time Imaging 2005; 11:429-42.

20. Wei GQ, Arbter K, Hirzinger G, editors. Automatic tracking of laparoscopic instrumenrs by color coding. Proceedings of the first international joint conference CRVMed-MRCAS 97 ; Grenoble, France; 1997.

21. Nishikawa A, Asano $S$, Fujica $R$, Yohda $T$, Miyazaki $F$, Sekimoto $M$, et al., ediwrs. Robust visual tracking of multiple surgical instrumenv for laparoscopic surgery. Proceedings of Computer Assisted Radiology and Surgery; London, UK; 2003.

22. Zhang $X L$, Payandeh S. Application of visual racking for robot-assisted laparoscopic surgery. J Robot Syst. 2002;19: 315-28.

23. Tonet $O$, Thoranaghatte RU, Megali G, Dario P. Tracking endoscopic instruments without a localizer: A shape-analysisbased approach. Comput Aided Surg. 2007;12:35-42.

24. Doignon C, Nageotte F, Maurin B, Krupa A. Pose estimation and feature cracking for robot assisted surgery with medical imaging. In: Kragic D, Kyrki V, editors. Unifying Perspectives in Computational and Robot Vision: Springer Verlag; 2007.

25. Climent J, Mares P. Automatic instrument localizarion in laparoscopic surgery. Electronic Lerrers on Computer Vision and Image Analysis. 2004;4:21-31.

26. Voros S, Long JA, Cinquin P. Automatic detection of instruments in laparoscopic images: A first scep towards high-level command of roboric endoscopic holders. Int J Rob Res. 2007; 26:1173-90.

27. Cano AM, Gaya F, Lamata P, Sanchez-Gonzalez P, Gomez EJ, ediwrs. Laparoscopic tool rracking method for augmenced realicy surgical applicarions. Proceedings of Internarional Symposium on Biomedical Simularion; London, $\mathrm{UK} ; 2008$. 
28. Sánchez-Margallo FM, Díaz-Guemes I, Pérez FJ, Sánchez MA, Loscertales B, Usón J. Preliminary results with a training program for thoracoscopic atrial fibrillation therapy. Surg Endosc. 2009;23:1882-6.

29. Usón J, Sánchez FM, Diaz-Guemes I, Loscertales B, Soria F, Pascual S. Animal models in urological laparoscopic training. Accas Urol Esp. 2006;30:443-50.

30. Bradski $G$, Kaehler A. Learning OpenCV. Computer Vision with the OpenCV Library. Loukides M, edimr. Sebastopol, CA, USA: O'Reilly; 2008.

31. Lucas $B$, Kanade $T$, editors. An Iteraive Image Registration Technique with an Applicarion to Scereo Vision. Proceedings of IJCAI; Vancouver, BC, Canada; 1981.
32. Bouguet J. Pyramidal implementarion of the Lucas Kanade feature tracker. Intel Corporation, Microprocessor Research Labs. 1999.

33. Freund $Y$, Schapire RE. A Decision-Theoreric Generaliztion of On-Line Leaming and an Applicacion w Boosring. Journal of Computer and System Sciences. 1997;55:119-39.

34. Viola $P$, Jones $M$. Robust real-time face detection. Int J Comput Vis. 2004;57:137-54.

35. Judkins TN, Oleynikov $D$, Stergiou $N$. Objecrive evaluacion of exper and novice performance during roboic surgical training tasks. Surg Endosc. 2009;23:590-7.

36. Mayhew J, Frisby J. 3D Model Recognirion From Sreteoscopic Cues. Cambridge, Massachusetts: MIT Press; 1991. 